\title{
CUIDADOS DE ENFERMAGEM A PESSOA IDOSA COM DEMÊNCIA DIANTE A PANDEMIA DA INFECÇÃO POR CORONAVÍRUS
}

Tânia Maria de Oliva Menezes'

ORCID: 0000-0001-5819-0570

Adriana Valéria da Silva Freitas'

ORCID: 0000-0003-1831-4537

Raúl Fernando Guerrero-Castañeda" ORCID: 0000-0003-3996-5208

Leidiane Nunes de Andrade ${ }^{\text {III }}$ ORCID: 0000-0003-1317-8986

'Universidade Federal da Bahia. Salvador, Bahia, Brasil.

"Universidad de Guanajuato. Celaya, Guanajuato, México.

I'Obras Sociais Irmã Dulce. Salvador, Bahia, Brasil.

Autor Correspondente: Tânia Maria de Oliva Menezes E-mail: tomenezes50@gmail.com

\section{INTRODUÇÃO}

A demência é um conjunto de manifestações neuropsiquiátricas que compõem um distúrbio, sendo sua característica essencial à deterioração das funções cognitivas e as mudanças de comportamento, sendo a Doença de Alzheimer (DA) a mais comum. É considerada uma desordem neurológica progressiva e irreversível, caracterizada pela perda progressiva da memória e pela funcionalidade prejudicada nos idosos; tem uma fase assintomática duradoura, passando por uma fase de pré-demência e finalmente estabelecendo a fase de demência ${ }^{(1)}$, exigindo atenção e cuidados diferenciados durante a pandemia da infecção por coronavírus.

Estima-se que a demência é a terceira causa entre as mortes em pessoas com mais de 65 anos de idade, gerando impacto na saúde pública ${ }^{(2)}$. O impacto da DA envolve, por um lado, o efeito sobre a qualidade geral de vida dos idosos e, por outro, o alto impacto que ela gera sobre a família e os cuidadores, dadas as características de deterioração progressiva. Exige continua informação a pessoa idosa, principalmente sobre importância do uso de máscaras, que nem sempre atendem as orientações fornecidas.

Atualmente, o mundo continua sendo afetado pela pandemia que gerou o SARS-CoV-2, mais conhecido como COVID-19, um tipo de coronavírus que gera uma síndrome respiratória aguda grave, neste momento, até mesmo pesquisas sobre os sintomas produziram novos dados. O que sabemos, é que a pessoa idosa é uma população em risco, sendo considerada vulnerável à COVID-19. Este grupo tem um risco maior de mortalidade de cerca de $15 \%$, além das comorbidades que apresentam, que aumentam o risco. A síndrome da fragilidade é um fator de ameaça importante para os idosos nessa condição ${ }^{(3)}$.

A pandemia afeta diretamente a pessoa idosa com as mais variadas demências, já que modificou os sistemas de saúde, a assistência médica e cuidados de enfermagem a pessoa idosa. Isso gerou um aumento da vulnerabilidade, já que, 
como mencionado, trata de um conjunto de determinantes: doença, acompanhamento médico, cuidadores, apoio familiar e social. Ademais, um paciente com doença mental pode ser mais suscetível a outros distúrbios que aumentam com o isolamento, confusão, raiva, ansiedade e agravamento de problemas de comportamento ${ }^{(5)}$.

Além desses problemas, os idosos com demências requerem um acompanhamento direto e próximo, que pode ser limitado; mas, também, dadas as características da condição, eles podem ter outras dificuldades relacionadas ao uso de Equipamentos de Proteção Individual (EPI), ou na compreensão das indicações do pessoal de saúde, e é claro que o cuidado dos idosos com demências é um cuidado muito específico, e é praticamente conflitante com o isolamento e distanciamento físico ${ }^{(4)}$.

Com relação aos idosos da comunidade e do lar familiar, a dinâmica da crise nos sistemas de saúde reduz o acompanhamento do tratamento e das consultas, ainda que isto seja considerado essencial ${ }^{(5)}$. Além disso, existem outras complicações, como o aumento da solidão devido ao isolamento, conflitos entre os cuidadores familiares, dependência econômica e as consequências disso.

A natureza inesperada da pandemia e a falta de clareza em seu escopo levaram a situações até um pouco inesperadas, como a modificação dos ambientes de atendimento, como nas Instituições de Longa Permanência para Idosos e seus recursos. Aqueles países onde já existe mobilidade de pessoas, mesmo com restrições, podem apresentar situações complexas.

A modificação das organizações que prestam cuidados e atenção somente aos idosos requeria atenção, tanto em uma esfera publica como privas como nos domicílios, o cuidado aos idosos com DA existem desafios importantes que não só impactam os idosos, mas, também, os cuidadores ${ }^{(6)}$. É imperativo pensar em diferentes cenários, já que a demência e seus cuidados devem buscar alternativas para promover a saúde mental do paciente e do cuidador, assim como o acompanhamento dos sintomas da demência, por outro lado, cada contexto no qual a pessoa idosa com demência está inserida deve se tornar um lugar seguro para suas atividades e para quem cuida.

A dinâmica das demências e DA no cuidado diário na família e nas instituições precisam ser redirecionadas, sendo importante rever as formas de cuidado para continuar o tratamento e, também, fornecer esse apoio aos idosos, à família e ao pessoal de saúde.

\section{OBJETIVO}

Descrever a experiência da enfermagem sobre os cuidados a pessoa idosa com demência diante a pandemia da infecção por coronavírus.

\section{MÉTODO}

Trata de um relato de experiência de enfermeiras sobre os cuidados a pessoa idosa com demência em diferentes contextos diante a pandemia da COVID-19. Para fundamentar o relato foi realizada busca da literatura de artigos científicos nacionais e internacionais, no ano de 2020, com os descritores demência, infecção por coronavírus e cuidados de enfermagem. Três eixos temáticos foram discutidos:

1. A pessoa idosa com demência que vive em Instituições de Longa Permanência para Idosos e a COVID-19;

2. A hospitalização da pessoa idosa com demência no contexto da infecção por coronavírus;

3. A pessoa idosa com demência no domicílio durante a pandemia.

\section{RESULTADOS}

\section{A pessoa idosa com demência que vive em Instituições de Longa Permanência para Idosos e a COVID-19}

Uma das preocupações dos especialistas em relação à institucionalização é a manutenção da qualidade de vida, autonomia e independência das pessoas idosas. Contudo, quando esse público possui alterações 
cognitivas e demências, essa preocupação é ainda maior. Pois se torna importante ofertar um ambiente propício para o desenvolvimento pessoal, tendo estímulo físico e mental que possa favorecer a manutenção de sua capacidade funcional(6).

A pandemia da COVID-19 trouxe para as Instituições de Longa Permanência para Idosos (ILPI's) o desafio de prevenir o contágio do vírus para os residentes. Essa tarefa se tornou ainda mais difícil quando os idosos possuem demência. Isso porque, uma das dificuldades que ocorre com a doença é a comunicação, levando a resistência dos idosos, principalmente relacionada aos cuidados diários e as medidas de isolamento.

A comunicação limitada pode ocorrer por problemas de memória, dificuldades sensoriais e cognitivas, interferindo na capacidade de interpretação, processamento e velocidade para responder aos estímulos. Isso torna a prestação dos cuidados diários desafiadores e mais exigentes, especialmente os cuidados de higiene ${ }^{(7)}$.

Nesse sentido, as orientações da equipe de enfermagem sobre a prevenção da higiene das mãos, uso do álcool em gel e de máscaras são muito importantes para manter os idosos mais protegidos, o que nem sempre são bem aceitas pelos mesmos, levando-os a retiradas das máscaras com frequência e agitação. Além disso, o distanciamento social, que também é uma das maneiras de prevenção contra a infecção do coronavírus, e importante no que diz respeito às pessoas externas à instituição, levou a proibição de visitas e desencadeou alterações de comportamento, levando a equipe de enfermagem a adoção de estratégias para minimizar essa condição, a exemplo das vídeochamadas.

Neste contexto, é preciso que a comunicação seja compreendida como uma ferramenta de cuidado importante, tanto quanto os demais cuidados, podendo ser capaz de colaborar no controle da agitação, fazendo com que o idoso aceite de maneira mais tranquila os cuidados implementados pelas instituições, e ofertados por cuidadores e profissionais de saúde. Esse cuidado muitas vezes é comprometido pelo reduzido número de profissionais nas ILPI's.

Dessa maneira, faz-se importante promover estratégias de comunicação eficientes, as quais precisam ser incluídas em programas de capacitação para que estejam mais próximos aos cuidados diários de idosos ${ }^{(8)}$. Assim, estes profissionais poderão compreender os comportamentos da pessoa idosa com demência, não reforçar a agitação e resistência a quaisquer cuidados que sejam oferecidos, principalmente no que diz respeito ao coronavírus, que ainda não possui vacinação, medida mais eficaz para o controle da infecção.

\section{A hospitalização da pessoa idosa com demência no contexto da infecção por coronavírus}

Para a pessoa idosa com demência, que necessita ser hospitalizada no contexto da pandemia, não é nada fácil, tanto para o paciente, quanto para a família e equipe que acompanha. A permanência da pessoa idosa durante a hospitalização pode suscitar conflitos de natureza física, social, mental e pessoal(9) $A$ mudança de ambiente e o confinamento no leito, ou num quarto, geram impactos negativos nesses idosos, visto que eles não têm cognição para entender o contexto de insegurança trazido nesse momento de pandemia e a real necessidade de estarem resguardados com as ações preventivas e de contenção social.

Não é fácil para a pessoa idosa com demência entender e assimilar a necessidade de se realizar a higienização das mãos, sempre que entrar em contato com ambientes e objetos externos. Eles também não compreendem a premência de se evitar tocar com as mãos no nariz, nos olhos e na boca, assim como não irão suportar o uso contínuo de máscara cirúrgica, devido ao desconforto que ocasiona e a sensação de aprisionamento da respiração. Porém, todas essas medidas são de suma importância na prevenção da contaminação pelo coronavírus.

Além disso, é imprescindível perceber os desafios que a pessoa idosa terá que enfrentar, ao pensar num cenário repleto de pessoas estranhas, todas paramentadas num ambiente com muito ruído, baixa temperatura, muita luminosidade e completamente diferente do ambiente de sua casa. Também, pela condição de serem obrigados a não estarem em companhia da família, devido ao risco deles se contaminarem no trajeto hospital/casa e se tornarem veículo de contaminação para a pessoa idosa. Nesse sentido, a orientação constante 
da equipe de enfermagem se faz necessária, para que a pessoa idosa com demência possa compreender as mudanças ocasionadas pela pandemia.

Todas essas condições são passíveis de gerar ansiedade, estresse e agitação psicomotora com perambulação, o que desencadeará alterações no padrão do sono e repouso, além de repercussões negativas na aceitação da dieta. Tudo isso, aumenta a predisposição da pessoa idosa desenvolver situações potenciais de risco como a ocorrência de quedas, maior risco de inatividade, lesões por pressão, incontinências, infecções relacionadas ao próprio ambiente hospitalar e os procedimentos invasivos. Além de quadros de desidratação, delirium e predisposição a fragilidade, devendo a equipe de enfermagem está atenta para prevenir complicações.

A equipe assistencial que trabalha com idosos com demência precisa ter o olhar sensível para perceber os fatores estressores, a exemplo de confinamento, isolamento dos laços culturais/religiosos/familiares, inatividade física, cognitiva e mudanças abruptos na rotina e no ambiente. Essa percepção possibilita a adoção de estratégias criativas e seguras para amenizá-los.

Dentre essas estratégias para reduzir o confinamento, a inatividade física e cognitiva dos idosos com demência no ambiente hospitalar pode-se citar a adoção de atividades lúdicas, itinerantes, individuais, de estimulo físico e cognitivo, realizada por uma equipe interdisciplinar. O planejamento dessas atividades deve levar em conta o grau de comprometimento cognitivo da pessoa idosa para a execução ocorrer da melhor forma possível e observando o distanciamento preconizado na pandemia.

Outra ação útil para diminuir a inatividade e quebrar o ambiente hostil do hospital é a criação de uma rotina de atividades individuais que ocupem o tempo ocioso, sendo que em tais atividades pode ser utilizada a música, que é um instrumento capaz de fazer o resgate cultural/social/religioso desses idosos. Além disso, acalma, alegra, descontrai o ambiente e pode diminuir o estresse e ajuda a passar o tempo na pandemia. Essa atividade pode ser feita, por exemplo, através do uso da música.

A atividade começa com o resgate temporal, no qual a equipe, ao entrar em contato com os idosos, questiona-os a respeito do mês, dia e ano, além de fazer um recorde temporal com os dias comemorativos e a alusão com as memórias guardadas por esses idosos de datas comemorativas culturais, sociais e religiosas. Esse carro poderá ser conduzido por um dos membros da equipe e passa por todas as enfermarias, de forma individual. Nesse tipo de atividade, também pode ser usado o som de voz e violão. Essas atividades devem adotar as medidas de segurança relacionadas à higienização das mãos, dos objetivos e utensílios, respeitar o distanciamento mínimo entre as pessoas e a restrição da quantidade de pessoas no local, para evitar aglomerações e o risco de contaminação pelo coronavírus.

Outra prática para atenuar o elemento estressor relacionado ao isolamento dos laços familiares é a adoção das visitas virtuais. Essas visitas são realizadas pelos profissionais da instituição, através de vídeo chamada pelo celular ou tablete institucional, pré-agendadas com os familiares e acompanhantes, ou pessoas eleitas como importantes para os idosos. Sendo assim, para acontecer à visita virtual, um trabalho interdisciplinar entre assistente social, psicologia, enfermagem e demais presentes na unidade durante a atividade. Precisa-se acompanhar e conduzir todo o processo, visto que é uma atividade que parece ser simples, mas, demanda tempo e gerenciamento das emoções suscitadas durante e após a atividade. Logo, as visitas virtuais são um instrumento útil e acolhedor, quando bem gerenciadas, nesse panorama da pandemia, além de uma maneira de aproximar paciente e família, num ambiente com segurança.

Infelizmente, a maioria dos serviços disponíveis no Brasil, para atender pessoas idosas com demência nessa conjuntura da pandemia, não estão preparados para responder as demandas necessárias para uma melhor assistência a essa clientela. Essas unidades, de modo geral, nem sempre dispõem de mão de obra especializada e com uma equipe interdisciplinar completa, e com o olhar sensível para a adequação dos fluxos de atendimento. Diante disso, essas unidades possuem um quantitativo de pessoal pequeno, com uma equipe mínima, muitas vezes, com pouca diversidade de profissionais, o que inviabiliza o atendimento de forma holística e multidimensional desses idosos, além desses serviços serem gerenciados por pessoas 
que não possuem conhecimento sobre as peculiaridades da pessoa idosa. Tal contexto contribui para uma assistência insegura e com desfechos negativos para a pessoa idosa com demência e sua família.

\section{A pessoa idosa com demência no domicílio durante a pandemia}

Idosos com demências no domicílio diante a pandemia da COVID-19 tiveram seus hábitos modificados, tendo em vista a recomendação do isolamento social. Ficar em casa levou a alterações de comportamento, agitação, agressividade, tentativa de fugir para a rua, apatia, hábitos noturnos que antes não existiam, entre outros. Estudo refere que a média de sintomas neuropsiquiátricos apresentados pelos idosos com demência e relatados pelos cuidadores investigados foram de quatro à cinco sintomas ${ }^{(10)}$. Se considerarmos o isolamento social, certamente esses sintomas tendem a serem exacerbados. Com isso, o desgaste de quem cuida se torna mais acentuado.

Outro aspecto importante se refere à presença do cuidador formal, quando já presentes em suas rotinas. Estabelecer fluxos de entrada e saída, como o banho e troca de roupa na chegada, com mudança de roupa e calçado; aferição de temperatura e uso de máscaras foram algumas mudanças necessárias a serem planejadas e orientadas pela enfermeira. Também, elaborar cronogramas de permanência do cuidador, para que eles possam ficar mais tempo no domicílio, uma vez que a escala variava de turnos de 12 até 24 horas, e com a pandemia, aumentar o tempo no domicílio é uma alternativa para reduzir a contaminação pelo vírus. Vale destacar que algumas famílias tiveram elevação dos custos com o cuidador, tendo em vista assumirem o deslocamento do cuidador de casa para o trabalho e vice-versa, seja com profissionais do trânsito, seja o próprio familiar, que se dispôs a esta tarefa, de maneira a proteger a pessoa idosa da contaminação pelo coronavírus.

Para a família cujo exercício profissional passou para o home office, houve dificuldades de conciliar a atividade laboral em casa, pois a pessoa idosa, a depender do estágio da demência, nem sempre compreende que não pode entrar no espaço destinado ao trabalho e, mesmo sendo orientado, ainda é possível gerar interrupções. Com isso, o desgaste com o cuidar se torna mais expressivo. Estudo refere sobre o desgaste do cuidador, devido à presença dos sintomas neuropsiquiátricos no idoso, sendo considerados muito desgastantes pelos cuidadores: o comportamento noturno $(61,9 \%)$, o comportamento motor aberrante $(58,4 \%)$, a irritabilidade $(47,3 \%)$, a desinibição $(47,1 \%)$, a agitação/agressividade $(47,0 \%)$ e a ansiedade $(45,8 \%)^{(10)}$. Com a identificação da elevação de alterações comportamentais na pessoa idosa com demências, cabe a equipe de enfermagem verificar as que mais se apresentam e orientar estratégias para minimizá-las.

É relevante considerar quando a pessoa idosa reside com netos, que deixaram de ir para escola e permanece todo o tempo em casa. Essa convivência mais intensa, que não acontecia anteriormente, é capaz de gerar mais conflitos, principalmente quando a demência se encontra mais avançada, e as alterações de comportamento são mais evidentes. A enfermeira pode dialogar com os netos, inclusive, informando sobre a demência e a necessidade de colaboração.

Mudanças nas atividades de vida diária também podem acontecer, a exemplo do banho e alimentação. Idosos com demências, que gritam quando não querem tomar banho, ou se alimentar, geram incômodos aos que residem no mesmo domicílio, que nem sempre compreende aquela condição. Com a pandemia, e não ter a possibilidade de sair como medida protetiva, as mudanças nessas atividades tem se destacado, remetendo a importância de orientações da equipe de enfermagem que favoreçam a aceitação da pessoa idosa.

A ausência de familiares que não moram no mesmo domicílio, e antes da pandemia se faziam presentes em visitas regulares, seja para almoços e/ou comemorações, também ocasionam ansiedade e alterações do comportamento. Apesar da demência, alguns familiares mais significativos para a pessoa idosa são lembrados, e passam a questionar com frequência onde estão e porque não aparecem. $O$ cuidado de enfermagem é sugerir como alternativa a vídeo chamada, especialmente quando a pessoa idosa apresenta ansiedade ou agressividade. Chamar aquele familiar que acalma é fundamental.

Um cuidado de enfermagem importante a destacar é o incentivo a pessoa idosa com demência a religiosidade não organizacional e espiritualidade, às vezes, único recurso valorizado e presente no cotidiano da pandemia. 
Também, se apresenta como alternativa recomendada para reduzir a exaustão de quem cuida. Estudo refere que a espiritualidade está vinculada ao enfrentamento para pessoa idosa diante de mudanças, sendo a fé e as orações um meio de acessar uma aproximação com o sagrado para proteção e apoio em situações adversas ${ }^{(11)}$, a exemplo do momento que vivem de pandemia, na qual houve intensas alterações em seus hábitos e rotinas.

\section{Contribuições para a Enfermagem}

As contribuições para a enfermagem estão em fornecer elementos para que possam prestar cuidados de enfermagem em intervenções presenciais e à distância, para que a pessoa idosa com demência melhor se adapte ao contexto da pandemia.

Além disso, as contribuições estão inseridas na possibilidade de ter um referencial que trata sobre o tema, contribuindo na implementação de cuidados de enfermagem alternativos que permite a assistência integral e individualizada, já que é a categoria profissional que está mais próxima à pessoa idosa com demência em diferentes contextos durante a pandemia da infecção por coronavírus.

\section{CONSIDERAÇÕES FINAIS}

A pandemia da COVID-19 exige esforços para os cuidados de enfermagem direcionados a pessoa idosa com demências nos cenários de Instituições de Longa Permanência para Idosos, hospital e domicílio para dirimir o impacto do isolamento social e de medidas preventivas nas alterações neuropsiquiátricas e evolução do quadro demencial. Contudo, em virtude das características da demência, torna-se importante uma visão holística do cuidado de enfermagem e acompanhamento à pessoa idosa e família na pandemia.

\section{AGRADECIMENTO}

Associação Brasileira de Enfermagem e Departamento Científico de Enfermagem Gerontológica - Nacional.

\section{REFERÊNCIAS}

4. Parnetti L, Chipi E, Salvadori N. et al. Prevalence and risk of progression of preclinical Alzheimer's disease stages: a systematic review and meta-analysis. Alz Res Therapy. 2019;11(7). https://doi.org/10.1186/s13195-018-0459-7

5. Garre-Olmo J. Epidemiología de la enfermedad de Alzheimer y otras demencias. Rev Neurol 2018;66:377-86. https://doi. org/10.33588/rn.6611.2017519

6. Morley JE, Vellas B. COVID-19 y adultos mayores. J Nutr Health Aging. 2020;24:364-5. https://doi.org/10.1007/ s12603-020-1349-9

7. El MH, Altintas E, Chapelet G, Kapogiannis D, Gallouj K. High depression and anxiety in people with Alzheimer's disease living in retirement homes during the covid-19 crisis. Psychiatry Res. 2020;291:113294. https://doi.org/10.1016/j. psychres.2020.113294

8. Greenberg NE, Wallick A, Brown LM. Impact of COVID-19 pandemic restrictions on community-dwelling caregivers and persons with dementia. Psychol Trauma. 2020;12(S1):S220-S221. https://doi.org/10.1037/tra0000793

9. Silva El, Lacerda TTB, Souza JA, Carvalho PF, Cássia Horta N, Souza MCMR. Avaliação da qualidade de vida do idoso institucionalizado com sinais de demência. Estud Interdiscip Envelhec. 2019;24(2):81-95. https://doi. org/10.22456/2316-2171.84716

10. Rosa C. Os cuidados de higiene corporal na pessoa idosa com demência [Dissertação] [Internet]. Lisboa: Escola Superior de Enfermagem de Lisboa; 2016[cited 2021 Jan 20]. Available from: http://hdl.handle.net/10400.26/19003

11. Bessa VFLM. A importância da comunicação no controlo da agitação em pessoas portadoras de demência-impacto de um programa educativo em cuidadores formais[Dissertação] [Internet]. Porto: FMUP; 2019 [cited 2021 Jan 20]. Available from: https://hdl.handle.net/10216/126585 
12. Lenardt MH. O cuidado de Enfermagem ao Idoso em Situação de Cronicidade e Hospitalização. In: Gonçalves LHT, Tourinho FSV. (Orgs). Enfermagem no Cuidado ao Idoso Hospitalizado. Barueri: Manole; 2012. p. 280.

13. Silva ILC, Lima GS, Storti LB. Sintomas neuropsiquiátricos de idosos com demência: repercussões para o cuidador familiar. Texto Contexto Enferm. 2018;27(3):e3530017. https://doi.org/10.1590/0104-07072018003530017

14. Gutz L, Camargo BV. Espiritualidade entre idosos mais velhos: um estudo de representações sociais. Rev Bras Geriat Gerontol. 2013;16(4):793-804. Available from: https://www.scielo.br/pdf/rbgg/v16n4/1809-9823-rbgg-16-04-00793.pdf 